\title{
p17 from HIV induces brain endothelial cell angiogenesis through EGFR-1-mediated cell signalling activation
}

\author{
Donghui Liu ${ }^{1,2} \cdot$ Yasmin Zeinolabediny ${ }^{1,2} \cdot$ Francesca Caccuri $^{3} \cdot$ Glenn Ferris $^{1} \cdot$ Wen-Hui Fang ${ }^{1} \cdot$ Ria Weston $^{1}$.

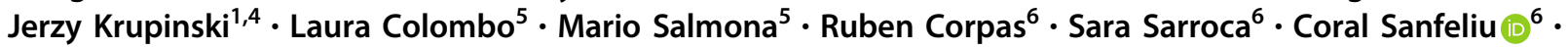 \\ Arnaldo Caruso $^{3} \cdot$ Baoqiang Guo $^{1} \cdot$ Xianwei Zeng $^{7} \cdot$ Mark Slevin $^{1,2,7}$
}

Received: 18 April 2018 / Revised: 28 August 2018 / Accepted: 29 August 2018 / Published online: 2 November 2018

(c) United States \& Canadian Academy of Pathology 2018

\begin{abstract}
HIV-associated neurocognitive disorder in HIV patients substantially reduces their quality of life. We previously showed that the HIV matrix protein, p17 could stimulate lymph-angiogenesis in vitro potentially contributing to lymphoma tumour growth and in addition is associated with vascular activation in neuro-degenerating brain tissue; here, therefore, we have investigated the detailed molecular mechanisms of this action. We performed in vitro cell culture, angiogenesis experiments, phospho-protein microarrays and Western blotting to identify cellular signalling induced by p17 within human brain endothelial cells (HbMEC), and inhibitor studies to block p17-induced vascular growth. We also characterised the effects of hippocampal CA1 injection of p17 on epidermal growth factor receptor-1 (EGFR1) expression linked to our murine model of dementia. p17 strongly induced angiogenesis of HbMEC (migration, tube formation and spheroid growth). p17 concomitantly increased phosphorylation of EGFR1 as well as down-stream intermediates ERK1/2, FAK, PLC- $\gamma$ and PKC- $\beta$ whilst an inhibitor peptide of EGFR, blocked cell signalling and angiogenesis. Finally, Mice that showed reduced cognitive function and behavioural deficiencies after p17 injection, demonstrated that p17 localised in cortical microvessels and also neurones many of which stained positive for p-EGFR1 by histology/IHC. This work provides strong support that p17 may be involved in initiating and/or perpetuating vascular tissue pathophysiology associated with comorbidity in HIV patients.
\end{abstract}

Electronic supplementary material The online version of this article (https://doi.org/10.1038/s41374-018-0147-z) contains supplementary material, which is available to authorized users.

Mark Slevin

m.a.slevin@mmu.ac.uk

1 School of Healthcare Science, John Dalton Building, Manchester Metropolitan University, Chester Street, Manchester M1 5GD, UK

2 University of Medicine and Pharmacy, Targu Mures, Romania

3 Department of Molecular and Translational Medicine, Section of Microbiology, University of Brescia Medical School, Brescia, Italy

4 Hospital Universitari Mútua de Terrassa, Department of Neurology, Terrassa, Barcelona, Spain

5 Department of Molecular Biochemistry and Pharmacology, IRCCS Istituto di Ricerche Farmacologiche "Mario Negri", Milan, Italy

6 Instituto De Investigaciones Biomedicas De Barcelona, CSIC, Barcelona, Spain

7 Weifang Medical University, Weifang, China

\section{Introduction}

Recent statistics show that still only 53\% of approximately 40 million people living with HIV receive antiretroviral therapy. The overall prevalence of major comorbidity varies between geographical location and quality of life, however, in spite of Highly Active Antiretroviral Therapy (HAART), it ranges between approximately $29 \%$ and $>60 \%$ (ages 18-49) [1-3] with health service associated costs running into the billions. For example, in Brent, London, UK, approximately $20 \%$ of cardiovascular disease (CVD) cases were diagnosed in people under the age of 49 with HIV, remaining significantly higher than the control population. Interestingly, however, the presence of comorbidities does not seem to be related to the time elapsed since diagnosis of HIV, suggesting a level of independency, and/or independent as yet un classified pre-determinates [2]. We propose that the protein 17 produced by HIV may have a causative role in accelerated progression of three of the major comorbidities those being neurocognitive decline, 
cancer, particularly lymphoma, and CVD with a specific relevance to its potent angiogenic capabilities $[4,5]$.

P17 is an HIV matrix protein that is critically involved in most stages of the retroviral life-cycle. In addition, p17 is a highly active signal transduction molecule and promotes activated T-cell secretion of cytokines [6]. It remains present in the circulation of HAART-treated patients [7] and can bind to CXCR1/2 receptors stimulating angiogenesis in endothelial cells (EC) and activation of $\mathrm{B}$ cell growth $[4,8,9]$. P17 is released in the extracellular space from HIV-1-infected cells and is easily detected in the plasma and tissue specimens [6, 10-12] of patients, including those successfully treated with HAART [10].

Disruption of neurocognitive functioning is one of the most frequent complications in patients infected with HIV. This is a common reason for consultation in HIV patients and it negatively affects their quality of life, treatment adherence and life span. Since the introduction of HAART, the spectrum of HIV-associated neurocognitive disorders (HAND) has been radically changed, with a significant reduction in dementia but a high prevalence of asymptomatic and mild neurocognitive impairments remains. Currently, in clinical practice, patients with HIV commonly experience memory, concentration or planification problems, even when virology is under control. These usually begin with subtle changes but it can lead to more severe forms of neurocognitive impairment. Before the introduction of HAART, HIV-associated neurocognitive impairment was considered a significant independent risk factor for death from AIDS. Later, it was shown that, although the median survival of patients with HAND has decreased considerably, it continues to be a negative prognostic factor associated with mortality [13, 14].

The pathological cause of cognitive decline in HIV patients is not fully understood; however [15], pathophysiological changes accompanying HAND include alterations in sphingolipid metabolism and in the frequency of viral astrocyte infection leading to glutamate accumulation and EC apoptosis, and dysfunctional autophagy of neurons [16]. In addition, monocyte activation, measured by microarray examination of gene profiles from HIV-positive patients, correlated with cognitive impairment, even in subjects controlled with HAART therapy [17]. HIVinduced signalling within the brain has been linked to neurotoxicity, most likely through deregulated GSK3 $\beta$ and CDK5 signalling pathways via cell surface receptors including CXCR4, NMDA and Notch1 [18]. Recently, Montoya et al. [19] showed increased expression of vascular endothelial cell growth factor, angiopoietin- 2 and Tie2 associated with arterial fibrosis, arterial stiffening and altered pulse pressure in patients with neurocognitive disturbance and HIV. Similarly, Yen et al. [20] showed a strong correlation between glomerular filtration rate and neurocognitive decline in HIV-positive individuals leading to a body of thinking around a direct relationship between vascular cognitive impairment associated to HIV disorder [21]. We previously showed that p17 from HIV caused dementia-like symptoms in a murine model following hippocampal injection [5]. In addition, there was a strong association between p17 staining and microvessel activation in human brain sections analysed by immunohistochemistry, suggesting a potential modulatory role in angiogenesis [8].

Extracellularly, p17 has been found to be a highly active signal transducing molecule capable of deregulating biological activities of many different cells that are directly involved in AIDS pathogenesis [9, 11, 22-29]. In particular, p17 is able to activate monocytes and T-cells by promoting the secretion of pro-inflammatory chemokines and cytokines [26-28]. Our unpublished data show that p17 has an important impact on coagulation and thrombosis, increasing vWF storage and secretion in EC, inducing plateletmonocyte aggregation and inducing coagulation and thrombosis directly in vivo (submitted elsewhere), therefore, a role for this protein in stimulation of early-onset and unstable CAD is likely.

The incidence of lymphoma in people with HIV is far greater $(66 \%$ higher) than that of the general population (1000-fold prior to HAART). Once again, the exact mechanisms responsible are not understood although immunosuppression and cytokine de-regulation, as well as the presence of the Epstein Barr infection, are thought to play an important role $[30,31]$. Post HAART, the incidence has fallen, particularly with long-term treatment, e.g., the incidence of aggressive B-cell lymphomas fell from $38 \%$ to $19 \%$ although this figure is still highly significant [32-36].

Mthembu et al. [37] demonstrated that aberrant angiogenic protein formation from mRNA gene splicing was associated with increased prevalence of Kaposi's sarcoma, cervical cancer and lymphoma, suggesting the importance of the vascular system in tumour growth. Recently, Giagulli et al. [38] showed that a single amino acid substitution in the p17 protein (making S75X) was able to predict B-cell activity and lymphomagenesis, and the same group showed previously that p17 stimulated lymphoma growth and metastasis through lymph-EC growth and activation via CXCR receptors and endothelin-1 [4].

In this study, we provide strong evidence that p17 protein may contribute to development of these major comorbidities in HIV-positive patients via induction of aberrant cell signalling, angiogenesis and vascular dysfunction and we defined an axis of the mechanism through activation of the epidermal growth factor receptor-1 (EGFR1). 


\section{Materials and methods}

\section{Recombinant proteins and antibodies}

Purified endotoxin (LPS)-free recombinant HIV-1 matrix protein p17 (in its monomeric form) was produced as previously described [29]. The absence of endotoxin contamination $(<0.25$ endotoxin units $/ \mathrm{ml})$ in the protein preparation was assessed by a Limulus amoebocyte assay (Associates of Cape Cod). The p17 mAb MBS-3 [10] was produced in our laboratory.

\section{Kinexus array and western blot analysis}

HbMEC were seeded in complete endothelial growth basal medium-2 (EBM-2) in a six-well plate at a cell concentration of $10^{5} / \mathrm{ml} /$ well and incubated in a water-saturated incubator at $37{ }^{\circ} \mathrm{C}$ and $5 \% \mathrm{CO}_{2}$. After $48 \mathrm{~h}$ incubation, the medium was renewed with serum poor medium (SPM, containing $0.5 \%$ FBS without growth factors) and cells incubated for further $24 \mathrm{~h}$ with or without EGFR neutralising antibody $(10 \mu \mathrm{g} / \mathrm{ml})$. Next, $5 \mu \mathrm{g} / \mathrm{ml} \mathrm{p} 17$ was added and the cells incubated for $8 \mathrm{~min}$ at $37^{\circ} \mathrm{C}$. Cells were lysed directly following removal of the medium with $120 \mu \mathrm{l} /$ well of ice-cold radioimmunoprecipitation (RIPA) buffer $(\mathrm{pH}$ 7.5) containing $25 \mathrm{mM}$ Tris- $\mathrm{HCl}, 150 \mathrm{mM} \mathrm{NaCl}, 0.5 \%$ sodium deoxycholate, $0.5 \%$ SDS, $1 \mathrm{mM}$ EDTA, $1 \mathrm{mM}$ sodium orthovanadate (EGTA), $1 \mathrm{mM}$ phenylmethylsulfonyl fluoride (PMSF), 1\% Triton X100 and $1 \mu \mathrm{M}$ leupeptin. The protein concentration of cell lysates was determined using the Bradford protein assay (Bio-rad, Munchen, Germany) and equal quantities of proteins (15 $\mu \mathrm{g})$ were mixed with $2 \times$ Laemmli sample buffer, boiled in a water bath for $15 \mathrm{~min}$ then centrifuged. Samples were separated along with pre-stained molecular weight markers (32,000-200,000 Da) by $12 \%$ SDS-PAGE. Proteins were electroblotted (Hoefer, Bucks, UK) onto nitrocellulose membranes (1 h) and the membranes were blocked for $1 \mathrm{~h}$ at room temperature in TBS-Tween ( $\mathrm{pH} 7.4$ ) containing $1 \%$ bovine serum albumin (BSA). Membranes were labelled with the following primary antibodies diluted in the blocking buffer, overnight at $4{ }^{\circ} \mathrm{C}$ on a rotating shaker: mouse monoclonal antibodies to phospho-PLC- $\gamma$, phospho-PKC $\beta 1$ and p-EGFR (Ty1173), rabbit polyclonal antibodies to p-FAK (Y397) p-ERK1/2; in this case, cells were cultured with p17 for $24 \mathrm{~h}$ ) and mouse monoclonal antibodies to $\beta$-tubulin $(1: 1000)$ from Santa Cruz Biotechnology. After washing $(5 \times 10 \mathrm{~min}$ in TBSTween at room temperature), membranes were incubated with either goat anti-rabbit or rabbit anti-mouse horse-radish peroxidase-conjugated secondary antibodies diluted in TBS-Tween containing 5\% de-fatted milk (1:1000, $1 \mathrm{~h}$, room temperature) with continuous mixing. After a further 5 washes in TBS-Tween, proteins were visualised using enhanced chemiluminescence (ECL) detection system (Geneflow). Experiments were repeated at least twice and a representative example is shown.

\section{Cell culture}

HbMECs were seeded in T75 flask pre-coated with $0.01 \%$ Poly-L-Lysine (Sigma, UK) and cultured in EBM-2, supplemented with growth factors and $2 \%$ foetal bovine serum (FBS) (Lonza, UK). At confluence, HbMECs were identified by their typical cobblestone morphology and "hill and valley" configuration, respectively. The cells used throughout the study were between passages 4 and 9. For inhibition assays, p17 protein/ and inhibitors were used/preincubated at the times and concentrations stated in the individual sections.

\section{Matrigel $^{\mathrm{TM}}$ endothelial tube formation assay}

The preparation of HbMECs was performed as described above. Briefly, $50 \mu$ l of Matrigel ${ }^{\mathrm{TM}}$ basement membrane Matrix reduced in growth factors (BD Bioscience, UK) was added to each well in 96-well plates and then incubated at $37^{\circ} \mathrm{C}$ for $60 \mathrm{~min}$ to let the Matrigel ${ }^{\mathrm{TM}}$ polymerise. After that, $9 \times 10^{3} / 50 \mu \mathrm{l}$ cells were added to each well including the conditions in low serum (0.5\% FBS) EBM-2 media with or without the scalar amount of tested compounds (FGF-2 at $250 \mathrm{ng} / \mathrm{ml}, \mathrm{p}-17$ at $50 \mu \mathrm{g} / \mathrm{ml}$ and EGFR neutralising antibody at $100 \mu \mathrm{g} / \mathrm{ml}$ ). Then the cells were incubated for $24 \mathrm{~h}$ at $37^{\circ} \mathrm{C}$. Controls were included in all experiments. After the 24 h-incubation, HbMECs migrated and aligned to form tubes (defined by the enclosure of circumscribed areas), a parameter of quantification. The number of closed tube-like structures was counted with a Zeiss phase contrast microscope. For the counting of enclosed areas, the cells were fixed with $4 \%$ PFA for $15 \mathrm{~min}$ and counts made in five fields by microscopy using the $\times 10$ objective. FGF-2 (25 $\mathrm{ng} / \mathrm{ml}$ ) was used as a positive control and EGFR-blocking peptide (Abcam ab2432; $10 \mu \mathrm{g} / \mathrm{ml}$ ) was incubated with the HbMEC cell mixture prior to plating to test the importance of the EGFR in P17-induced angiogenic signalling. Experiments were repeated three times, each from three wells of a 96-well plate and results are shown as mean \pm SD.

\section{Spheroids sprout assay method}

HbMEC cells were maintained in EBM-2 medium (Lonza, UK) containing $2 \% \mathrm{FBS}$ and growth factors. The cells were starved (in $0.5 \%$ FBS) $24 \mathrm{~h}$.

After the initial trypsinisation stage, cells $\left(6 \times 10^{5}\right.$ cells $/ \mathrm{ml}$ suspended in EBM-2 medium) were added to non-adhesive 96-well Greiner ${ }^{\circledR}$ plates (Sigma, UK) for suspension 
culture. Firstly, $3 \mathrm{ml}$ of methylcellulose was made up with $15 \mathrm{ml}$ of EBM-2 medium, from this $15 \mathrm{ml}$ were added to $250 \mu \mathrm{l}$ of the initial cell volume. The cells ( $150 \mu \mathrm{l} /$ well) were then added to the 96-well plates and incubated in a humidified $5 \% \mathrm{CO}_{2}$ incubator at $37^{\circ} \mathrm{C}$ for $24 \mathrm{~h}$.

In a separate bijou, $1.33 \mathrm{ml}$ of collagen gel solution (Sigma UK) at $(2 \mu \mathrm{g} / \mathrm{ml})$ was added to $920 \mu \mathrm{l}$ of EBM-2 medium and kept on ice. Before adding the spheroids in the 24-well plate, the collagen solution was neutralised by adding $150 \mu \mathrm{l} 0.1 \mathrm{~N} \mathrm{NaOH}$. The spheroids were collected, added to $15 \mathrm{ml}$ tubes and centrifuged at $400 \times g$ for $3 \mathrm{~min}$. When a clear pellet was distinguished, the supernatant was removed and the pellet was kept in a volume of about $112.5 \mu \mathrm{l}$ per tube, $25 \mu \mathrm{l}$ of conditions (FGF-2 at $250 \mathrm{ng} / \mathrm{ml}$, p-17 at $50 \mu \mathrm{g} / \mathrm{ml}$ and EGFR neutralising antibody at $100 \mu \mathrm{g} /$ $\mathrm{ml}$ ) and $112.5 \mu \mathrm{l}$ methylcellulose gel was added, then mixed well with gentle pipetting. The conditions were added, and SPM [0.5\% (v/v) FBS EBM-2] was applied to the cells. The spheroids were then added to the 24-well plates at $500 \mu \mathrm{l} /$ well. They were incubated in a humidified $5 \% \mathrm{CO}_{2}$ incubator at $37^{\circ} \mathrm{C}$. After $24 \mathrm{~h}$, the spheroids were fixed with $4 \%(\mathrm{w} / \mathrm{v})$ paraformaldehyde (PFA) for $15 \mathrm{~min}$ at room temperature and pictures were taken. Note: Our pilot data and that of others indicates that spheroids prepared in this way can continue to grow over periods of $72 \mathrm{~h}$ and beyond, however the complications of cell division and integration into tubes over time determined our optimal time of incubation to be $24 \mathrm{~h}$ when the impact of migration and sprouting alone can be measured most accurately. Sprouting occurred from the spheroid core, and the sprout length (mean \pm SD) was estimated with the software Image-J using five spheroids with similar sizes of the core of spheroid from three wells of the plate. Representative examples are shown in the results.

\section{EC migration assay}

HbMEC were seeded respectively on $1 \mathrm{~mm} \times 1 \mathrm{~mm}$ glass cover slips at a concentration of $4 \times 10^{5}$ cells $/ \mathrm{ml}$ in $1 \mathrm{ml}$ of completed EBM-2 medium in each well of a poly-L-lysine pre-coated 12-well plates and incubated in a water-saturated incubator at $37{ }^{\circ} \mathrm{C}$ and $5 \% \mathrm{CO}_{2}$. When cells reached about $80 \%$ confluence, the growth medium was replaced with serum poor medium [SPM, basal EBM-2 medium containing $0.5 \%(\mathrm{v} / \mathrm{v}) \mathrm{FBS}$ ] and incubated for a further $24 \mathrm{~h}$. After $24 \mathrm{~h}$ of humidified incubation at $37^{\circ} \mathrm{C}$ in $5 \% \mathrm{CO}_{2}$, each well of the 12-well plates was washed with warm PBS three times and the adherent cells were then scratched in one single continuous line across the glass cover slip using a razor blade and the wells were then washed carefully with warm PBS three times to remove any floating cells. Then conditions (FGF-2 at $25 \mathrm{ng} / \mathrm{ml}, \mathrm{p}-17$ at $5 \mu \mathrm{g} / \mathrm{ml}$ and EGFR neutralising antibody at $10 \mu \mathrm{g} / \mathrm{ml}$ ) were added, and SPM [0.5\% (v/v) FBS EBM-2] was applied to the cells, then the cells were incubated under the same conditions as mentioned above for $24 \mathrm{~h}$.

After the $24 \mathrm{~h}$ incubation, $100 \mu \mathrm{l}$ of $4 \%$ (w/v) PFA was added to each well to fix the cells at room temperature (RT) for $15 \mathrm{~min}$. The medium was then removed, and the wells were washed with PBS. $100 \mu$ of $100 \%$ ethanol was added to the cells and left for $5 \mathrm{~min}$. Then the ethanol was removed, and the wells were left to dry before staining the cells with methylene blue for $5 \mathrm{~min}$. The stain was removed, and the wells were washed with distilled water $\left(\mathrm{dH}_{2} \mathrm{O}\right)$. Finally, the cell migration was assessed by phase contrast microscopy and images were taken using a digital camera (Zeiss). In this experiment, cells were treated in triplicate for each experimental condition and pictures of five areas of each well were taken. The picture analysis was performed using Image-J. Both migration distance and number of migrated cells were measured and the mean \pm SD are given. Each experiment was performed three times and representative example is given.

\section{Immunohistochemistry and immunofluorescent staining}

Immunohistological analysis was carried out on murine brains following intra-hippocampal injection of p17 as described in our previously published work [5] to determine the localisation of $\mathrm{p} 17$. Brains, fixed in $4 \%$ paraformaldehyde in $100 \mathrm{mM} \mathrm{PB}$ and paraffin, were processed according to standard histological protocols $(n=5$ mice were used that were p17-injected; 10 sections from each analysed). Sections were incubated at room temperature over night with the monoclonal anti-p17 MBS-3 antibody (1:100 vol/vol), and/or anti-phospho-EGFR1 antibody (Abcam 5644, Y1068), sections were washed three times with $10 \mathrm{mM}$ PBS, pH 7.4 and then incubated for $1 \mathrm{~h}$ with the appropriate secondary antibodies: rabbit anti-mouse biotinylated (Vector Labs; 1:500 dilution vol/vol), 3,3' diaminobenzidine (DAB, Vector Labs, UK) or Nickel DAB (N-DAB, Vector Labs, UK) for immunohistochemistry. Images were captured with Nikon 80i Digital Microscope using Nis Elements 3.21 software with multichannel capture option. Negative control slides were included where the primary antibody was replaced with 5 mM PBS, pH 7.4.

\section{Statistical analysis}

Data are presented as the mean \pm SD. Statistical analysis was performed using GraphPad Prism software version 7.0 for Windows (GraphPad Software). The values were compared using paired Student's $t$-test or non-parametric 
Wilcoxon test. The differences at $p<0.05$ were considered statistically significant.

\section{Results}

\section{P17 stimulated angiogenesis in HbMEC}

P17 $(5 \mu \mathrm{g} / \mathrm{ml})$ stimulated spheroid sprout formation significantly $(*)$ increasing both the number and length of sprouts compared to the control (Fig. 1a). Figure 1b shows that when 2 spheroids are placed adjacent to each other, p17 has the capability to induce chemotactic response as shown by polarised directional movement of cells (arrow). In comparison FGF-2 produced a less "aggressive" nonepolarised movement of cells from one spheroid to another. Regarding migration (in a scratch wound assay) p17 stimulated cell movement into the denuded area to a similar degree as the FGF-2 positive control after $24 \mathrm{~h}$ incubation (Fig. 1c). Note the directional 'streaming' of cells in wounds induced by p17 (arrows). Tube-likestructure formation was significantly stimulated by p17 (5 $\mu \mathrm{g} / \mathrm{ml} ; 24 \mathrm{~h}$ ) with tubes similar in size and thickness to those formed using the positive control FGF-2 $(25 \mathrm{ng} / \mathrm{ml}$;
Fig. 1d). With such a strong activation of angiogenesis we attempted to dissect the possible mechanisms through which HbMEC were stimulated. All experiments were performed at least twice in triplicate and a representative example is shown. * indicates $p<0.05$ and significance.

\section{p17 induced HbMEC angiogenic signal transduction activation/protein phosphorylation through EGFR1}

Kinexus phospho-protein-array analysis $\left(\mathrm{KAM}^{\mathrm{TM}} 1.2\right.$ 500), carried out using HbMEC exposed to p17 for $8 \mathrm{~min}$ identified increased EGFR1 phosphorylation at amino acid residue $\mathrm{Y} 1172$ in addition to other protein phosphoupregulation that is associated with angiogenesis (e.g., MEK/ERK and PLC- $\gamma 1$; Table 1). Western blotting confirmed upregulation of EGFR-1-Y1172, ERK1/2 and PLC- $\gamma 1$ and also the upstream protein FAK, strongly associated with EC migration through Rho GTPases. Importantly, a specific blocking peptide (Abcam-2432/ $10 \mu \mathrm{g} / \mathrm{ml}$ ) was able to significantly perturb EGFR 1 phosphorylation by p17 Fig. 2a), and in addition, downstream cell signalling via PLC $\gamma 1$, PKC- $\beta$ and ERK1/2, whilst having a weaker/or no notable inhibitory effect on FGF-2-induced phospho-protein signalling (Fig. 2a). For
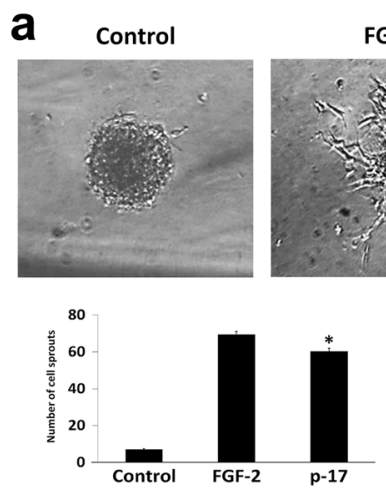

FGF-2
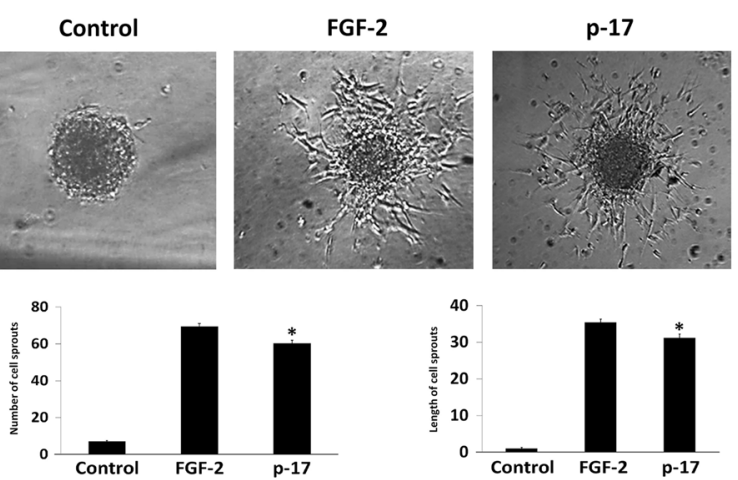

C

Control
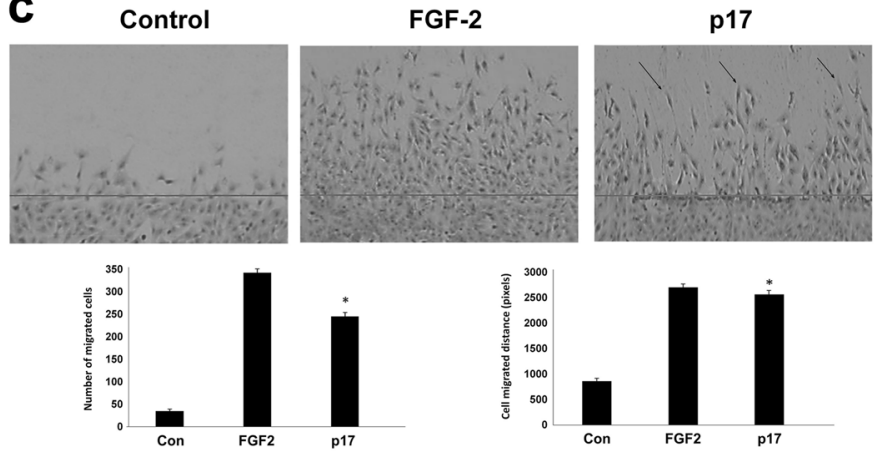

Fig. 1 a p17 stimulates HbMEC spheroids. b p17 induces HbMEC spheroid directional sprouting and chemotaxis attraction to each other. c p17 promotes HbMEC migration. d p17 promotes HbMEC tube formation. HbMEC treated with FGF-2 $25 \mathrm{ng} / \mathrm{ml}$ were used as positive
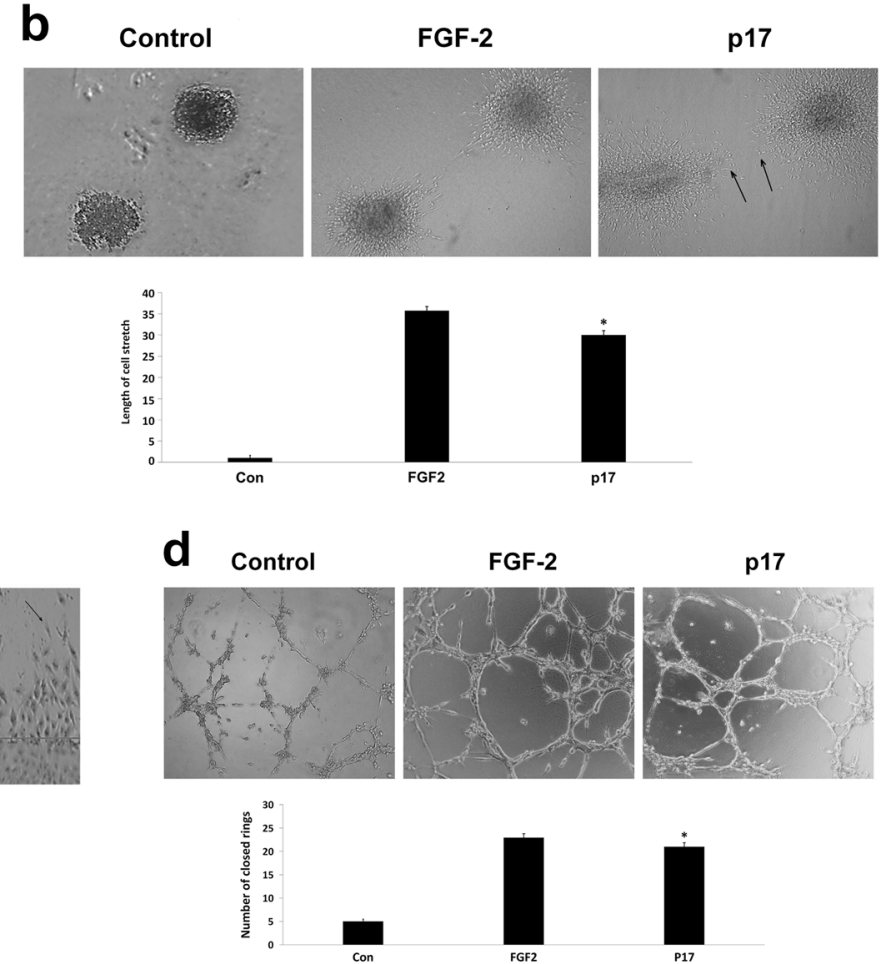

control. Each test was conducted in triplicate and statistical analysis performed using the Wilcoxon matched pair test. The bar chart represents the relative angiogenic effect compared with control cells 


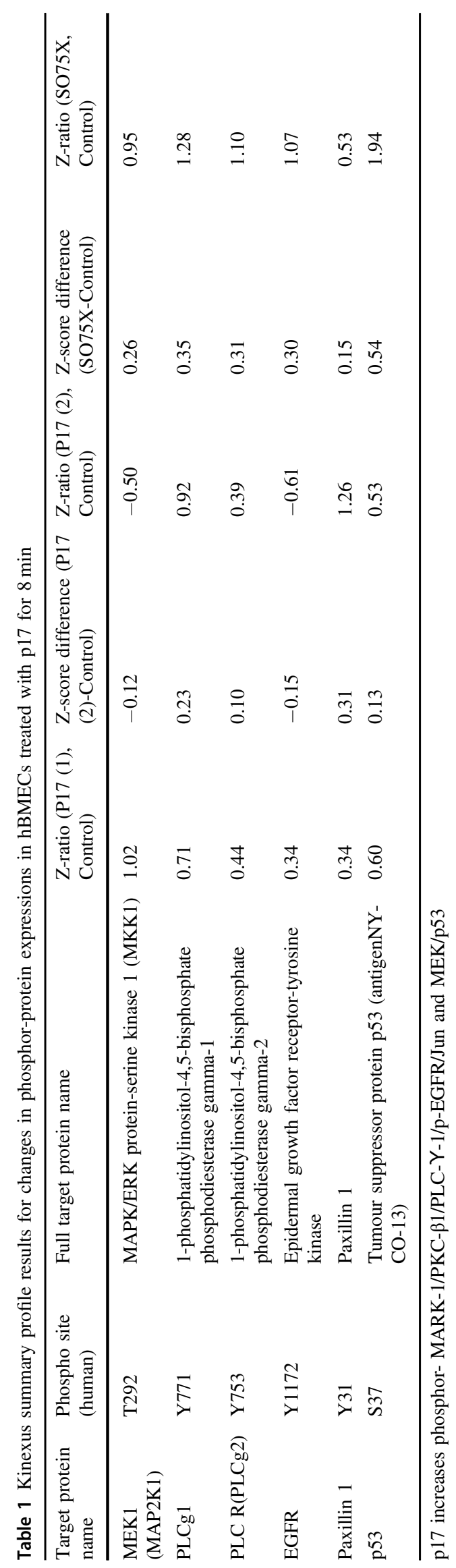

an unknown reason, FAK-phosphorylation was increased beyond control basal levels in the presence of the EGFR inhibitory peptide. All experiments were performed at least twice in triplicate and a representative example is shown.

\section{HbMEC angiogenesis was dependent on EGFR signalling}

Pre-incubation with the EGFR-inhibitor peptide blocked HbMEC angiogenesis, inhibiting significantly, p17 (5 $\mu \mathrm{g} /$ ml)-induced spheroid sprout formation (Fig. 3a), scratchwound healing (Fig. 3b), and tube-like-structure formation (Fig. 3c), and whilst only weakly and none-significantly affecting FGF-2-induced angiogenesis (except in the case of sprouting from spheroids where FGF-2 induced sprouting was impaired by around 25\%). All experiments were performed at least twice in triplicate and a representative example is shown. * indicates $p<0.05$ and significance.

\section{EGFR was overexpressed in EC in mice following hippocampal injection of $\mathbf{p} 17$}

Mice, euthanized after 4 months following stereotactic administration of p17 into the hippocampalCA1 region, had the brains removed and histological sections cut all the way through transversely. Here, we show by immunohistochemistry examination of $n=5$ animals that injection of p17 caused a notable increase in the presence of phosphorylated EGFR1 in local cortical microvessels and colocalised with p17 (DAB brown-EGFR1 and Nickel DABp17) (i) at $\times 40$ and (ii) at $\times 200$ ). Local cortical neurones also showed cytoplasmic staining of the EGFR1 (iii) and some hippocampal neurones (iv; arrows) (Fig. 4). Staining for EGFR1 (phospho) was not seen in sham-operated untreated animals (v) and (vi) shows a control IHC with the primary antibody replaced by PBS. For a detailed assessment of the IHC and histology as well as cognitive and behavioural profiles from these animals and experiments, where we show that injection of the protein caused significant cognitive impairement, please see Zeinolabediny et al. [5]. Note: data showing the neurocognitive impact of the injection of p17 protein into the mouse hippocampus are shown for convenience as Supplementary Figures 1 and 2.

\section{Discussion}

We previously demonstrated that p17 from HIV can be found within the brain of HIV-infected patients with dementia, and in addition, we have considerable evidence supporting potent biological properties, stimulating 
Fig. 2 a Western blotting demonstrated p17-induced phosphorylation of angiogenic signalling intermediate's in HbMEC. The figure also shows increased expression of $\mathrm{p}$ EGFR, p-ERK1/2, p-PLC $\gamma 1$, $\mathrm{p}-\mathrm{PKC} \beta 1$ and $\mathrm{p}-\mathrm{FAK}$ in p17-treated cells (8 min), notably reduced in the presence of the EGFR-peptide antagonist. Tubulin was as loading control. All experiments were repeated at least twice and a representative example is shown

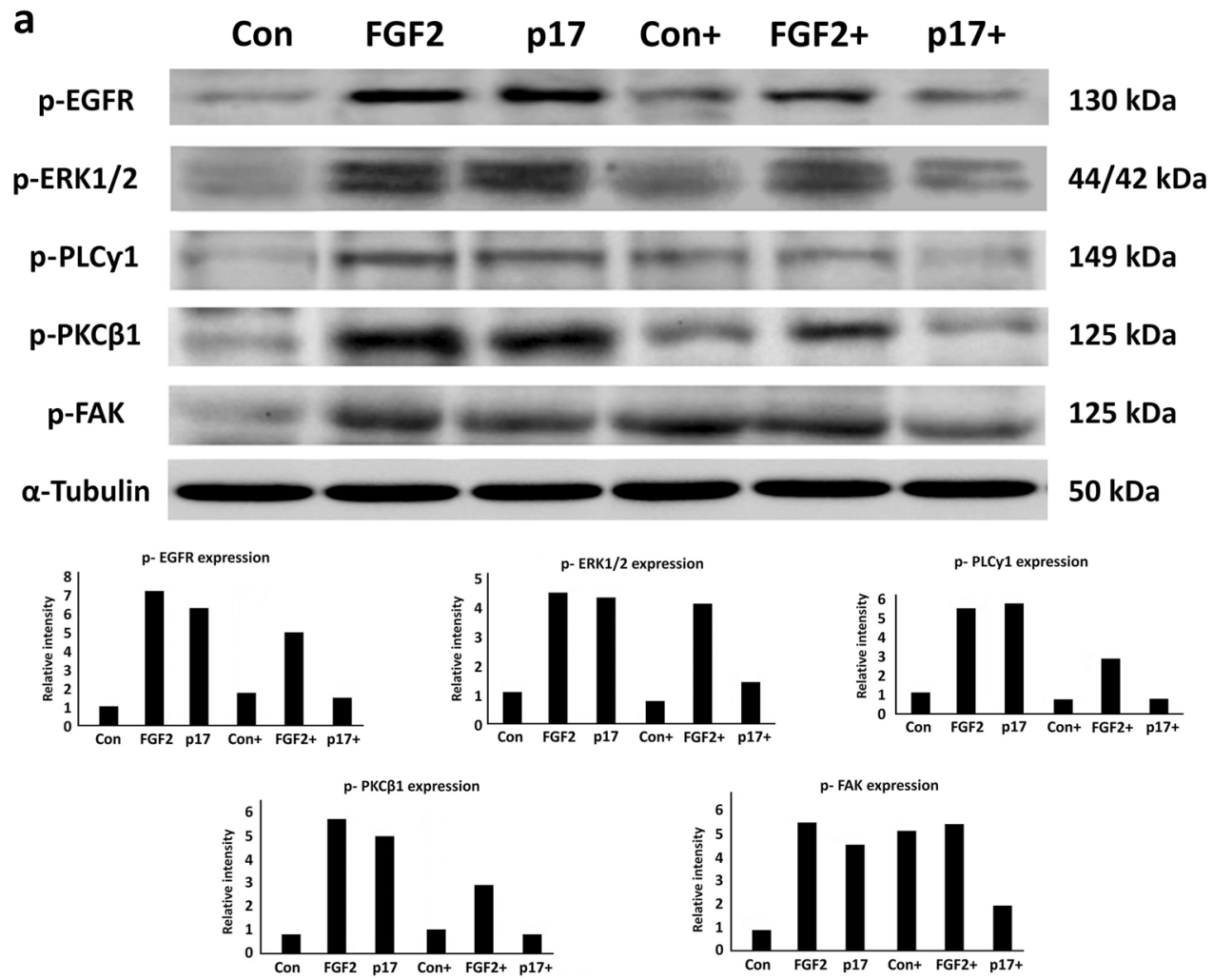

Con: Control; FGF2: ECs treated with FGF2 as positive control; p17: ECs treated with p17; Con+: ECs treated with EGFR neutralise blocker; FGF+: ECs treated with FGF2 and EGFR blocker; p17+: ECs treated with p17 and EGFR blocker lymphangiogenesis and B-cell activation in vitro [7, 9, 39]. Here, we show a clear role for $\mathrm{p} 17$ in the promotion of vascular activation and detail the p17-intracellular signalling pathway operating through EGFR whilst identifying a potential orphan drug that could perturb its aberrant biological properties.

In reference to angiogenesis, considering our previous finding that cortical microvessels of p17-hippocampal injected mice retain and express p17 concomitant with CD105 (a marker of activation and angiogenesis in EC), our in vitro experiments went on to investigate how p17 might influence the angiogenic process.

P17 strongly stimulated migration, sprouting, chemotaxis and tube-like-structure formation in HbMEC, with a similar potency to FGF-2 a known angiogenic mitogen. So far, the pathobiological and physiological relevance of activating brain microvessels and its impact upon a possible neurodegenerative process is not known. However, excessive improper vascularisation could result in non-patent vasculature, haemorrhage and neuronal hypoxia through abnormal remodelling [40]. In addition, vascular activation is now considered to be a critical process in the establishment of neurodegenerative progression. For example, in two separate transgenic murine models of AD, the cerebro-vasculature was studied in detail and was found to be activated with overexpression of amyloid beta, thrombin, tumour necrosis factor alpha, interleukin-1 beta, interleukin-6 and matrix metalloproteinase-9. Drug inhibition (sunitinib) significantly reduced vascular expression of these proteins, concomitantly improving cognitive function and reducing associated brain inflammation [41].

Aberrant EGFR signalling could predispose brain or other microvessels towards accelerated vascular angiogenesis and dysfunction, and has been identified recently as part of a network cluster within the CSF and plasma proteome-as a significant gene associated with AD risk [42]. In addition, EGFR-A $\beta$ fusion constructs expression dramatically enhanced neuronal cell death via ASK1/JNK signalling pathways implicating it in a role under these circumstances linked to neurodegeneration [43]. A $\beta$ also induced apoptosis of rat PC12 cells via FAK stimulation through NF-kB and ERK1/2 [44], suggesting this pathway activation through p17 could damage the hippocampal and cortical neurons we observed staining positive, although these processes require further detailed investigation.

An in-direct role for HIV has been postulated in driving HIV-associated lymphoma the disease via enhanced cytokine production and loss of immune function, since the virus does not enter into the genome of B-cells [45]. However, the importance of lymphangiogenesis in sustaining growth and metastasis of the tumours is critical [46], and we previously 

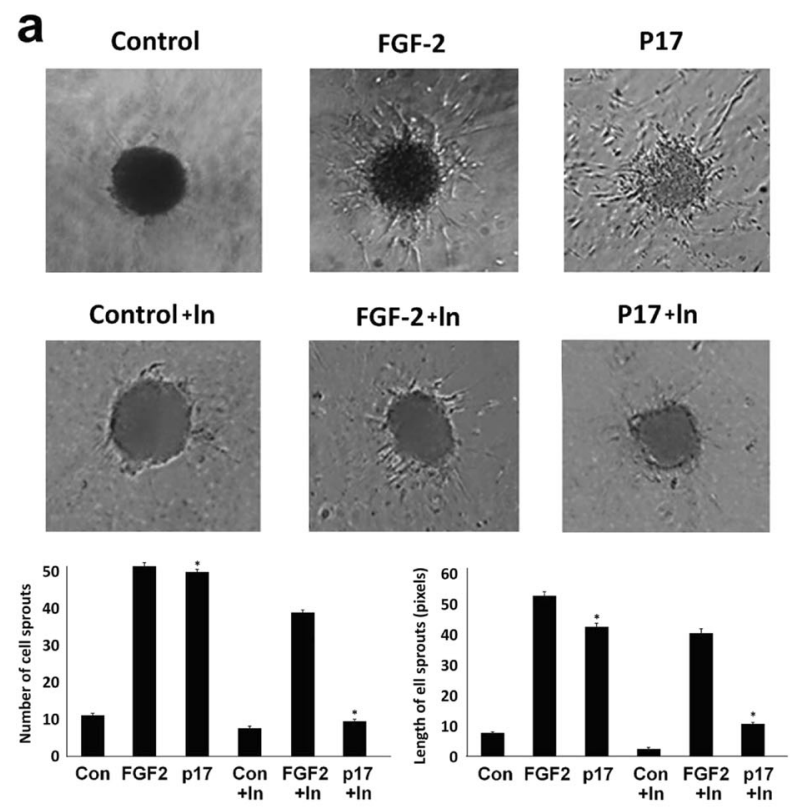

b

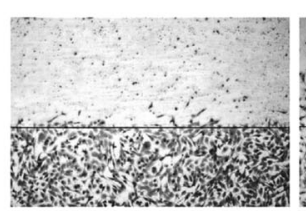

Con+ln
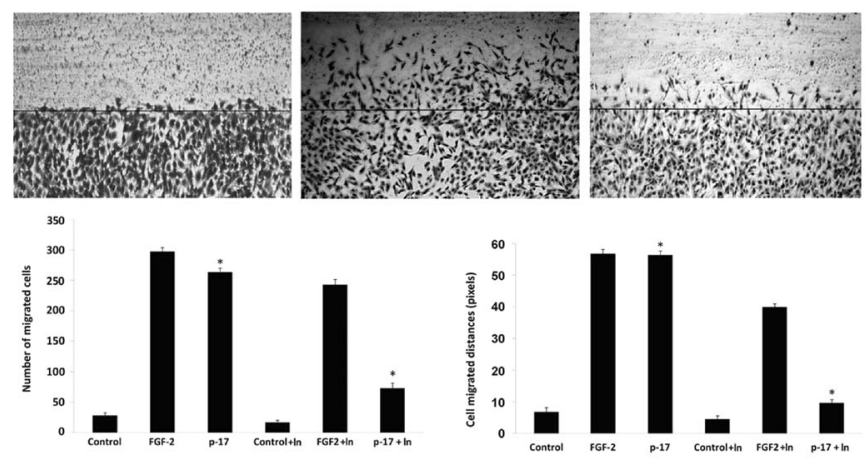

FGF-2

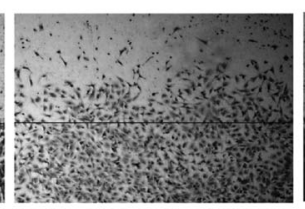

FGF2+In

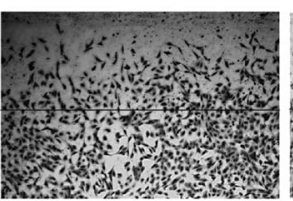

P17+ln

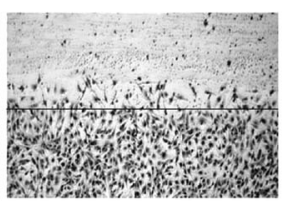

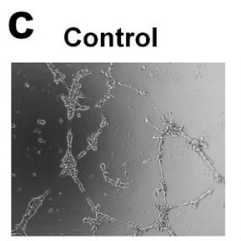

FGF-2
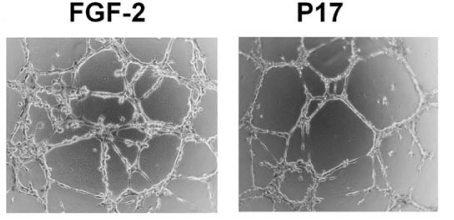

Control
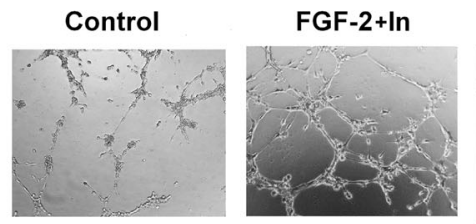

P17+ln
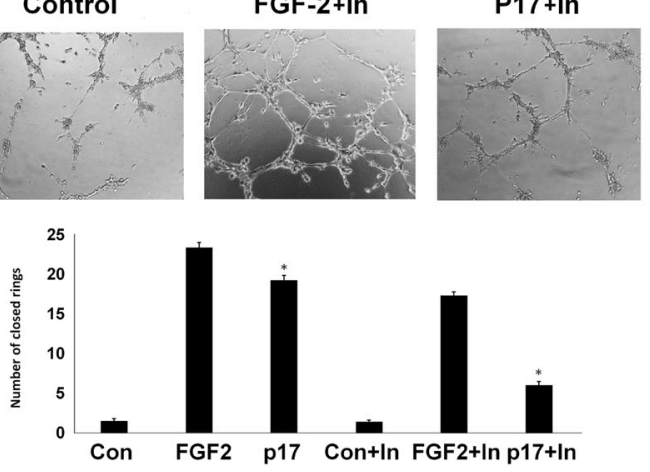

Fig. 3 a HbMEC spheroids sprouting was significantly blocked by the EGFR neutralising receptor antagonist in p17-treated cells. b p17-promoted HbMEC migration and this was significantly reduced in the presence of the EGFR antagonist. c p17-promoted HbMEC tube formation was blocked by the EGFR antagonist. HbMEC treated with

FGF-2 $25 \mathrm{ng} / \mathrm{ml}$ were used as positive control. The bar chart represents the relative changes compared with control cells. Each test was conducted in triplicate and statistical analysis performed using the Wilcoxon matched pair test. Experiments were repeated at least twice and a representative example is given

showed that p17 was capable of stimulating the production of several angiogenic growth factors including endothelin-1 and inducing lymphatic EC growth through an autophagy-based pathway [47]. Here, we have performed a detailed study on HbMEC and shown that p17 stimulates angiogenesis through the EGFR1 and this may be a critical component of T-cell metastasis through Rac-1 signalling [48].

There are several studies now strongly implicating 'unstable' atherosclerotic plaque thrombosis and aberrant or enhanced angiogenesis in combination with sheer stress or other risk factors [49]. Cases of acute coronary thrombosis in HIV positive individuals also occur often at young ages [50]. Concurrent activation of platelets, monocytes and EC by p17 from HIV,most certainly promotes a thrombotic inflammatory environment that might pre-dispose these individuals to early onset AMI [51]. Hence, previous findings of the pro-monocytic activation of p17 [52], platelet aggregating and tissue factor stimulation (submitted elsewhere) and here, angiogenic capabilities, indicate a mechanism through which this co-morbidity could develop.

We provide data here showing that direct inhibition of the EGFR1 using a blocking peptide is sufficient to abrogate 

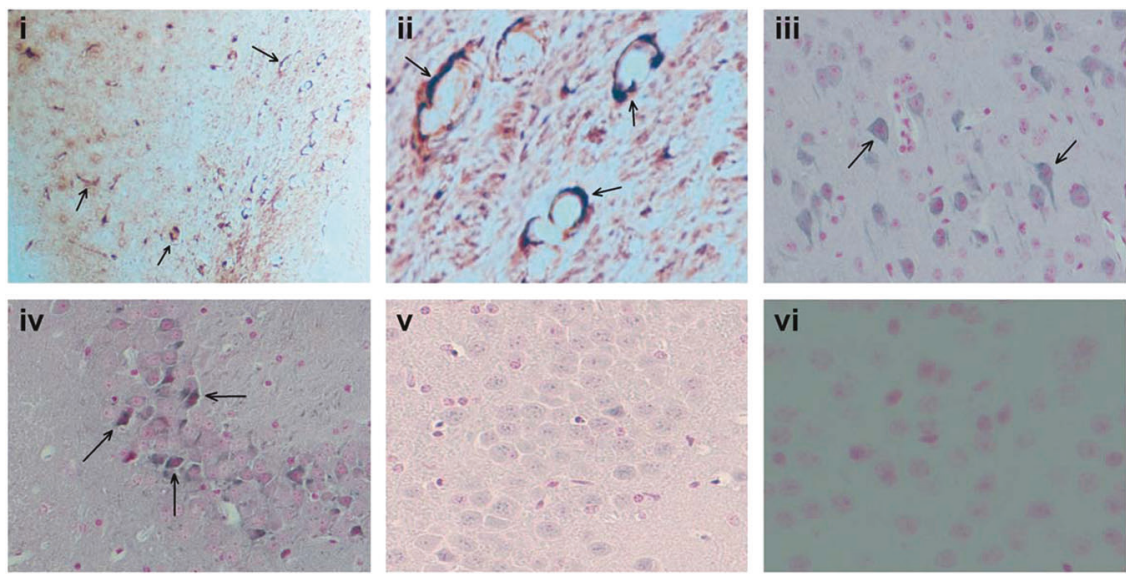

Fig. 4 Histology/IHC of p17 in the CA1 hippocampus and local cortical regions. Showed that EGFR1 was observed in local cortical microvessels (i $\times 40$ and ii $\times 200$; Nickel DAB and p17-DAB brown; arrows). (iii) Shows cytoplasmic localisation of EGFR1 in cortical neurones adjacent to the p17 injection point (Nickel-DAB; $\times 400)$. And iv, shows EGFR1 expression in local hippocampal neurones (arrows; vector blue $\times 400$ ). (v) Shows non-injected control hippocampal region with negative EGFR1 staining whilst vi, shows control IHC with the primary antibody replaced by PBS. In all 10 sections from five p17-injected murine brains were analysed together with 3 control mice angiogenesis mediated by $\mathrm{p} 17$. So far we are not sure of the exact mechanism (specific interactions between p17 and the EGFR/EGF or other) but this provides evidence demonstrating a critical interaction via the EGFR/EGF axis and a potential therapeutic opportunity for prevention of p17-induced HIV complications.

In conclusion, we have identified a critical pathway of p17 action through stimulation of EGFR signalling that may contribute to comorbidity development in HIV patients. What the potential implications of these findings may be when linked to cognitive decline/dementia and other comorbidities such as lymphoma development and aggression is not sure at this time. However, stimulation of angiogenesis /lymph angiogenesis through EGFR activation could help us to understand how lymphoma and possibly other tumours are initiated or perpetuated in HIV-positive individuals. In HAND, we are still not sure of the role (if any) of p17 in activation or control of neuronal or glial behaviour-we could postulate that abnormal vascularisation as shown in this work could impact on vascular types of dementia but this also remains to be proven.

\section{Compliance with ethical standards}

Conflict of interest The authors declare that they have no conflict of interest.

\section{References}

1. Goulet JL, Fultz SL, Rimland D, Adeel Butt, Cynthia Gibert, et al. Do patterns of comorbidity vary by HIV status, age, and HIV severity? Clin Infect Dis. 2007;45:1593-601.
2. Lorenc A, Banarsee R, Robinson N. Complementary therapy provision in a London community clinic for people living with HIV/AIDS: a case study. Complement Ther Clin Prac.. 2014;20:65-69.

3. Zigmond T. Mental health law across the UK. BJPsych Bull. 2017;41:305-7.

4. Caccuri F, Rueckert C, Giagulli C, Schulze K, Basta D, Zicari S, et al. HIV-1 matrix protein 17 promotes lymphangiogenesis and activates the endothelin-1/endothelin B receptor axis. ATVB.. 2014;34:846-56.

5. Zeinolabediny Y, Caccuri F, Colombo L, Morelli F, Romeo M, Rossi A, et al. HIV-1 matrix protein p17 misfolding forms toxic amyloidogenic assemblies that induce neurocognitive disorders. Sci Rep. 2017;7:10313.

6. Fiorentini S, Marini E, Caracciolo S, Caruso A. Functions of the HIV-1 matrix proteinp17. Microbiol-QJ Microbiol Sci.. 2006;29:1-10.

7. Giombini E, Dolcetti R, Caccuri F, Selleri M, Rozera G, Abbate I, et al. Detection of HIV-1 matrix protein 17 quasispecies variants in plasma of chronic HIV-1-infected patients by ultra-deep pyrosequencing. JAIDS.. 2014;66:332-9.

8. Caccuri F, Giagulli C, Bugatti A, Benetti Anna, Alessandri Giulio, Ribatti Domenico, et al. HIV-1 matrix protein p17 promotes angiogenesis via chemokine receptors CXCR1 and CXCR2. Proc Natl Acad Sci USA. 2012;109:14580-5.

9. Caccuri F, Giagulli C, Reichelt J, Martorelli D, Marsico S, Bugatti A, et al. Simian immunodeficiency virus and human immunodeficiency virus type 1 matrix proteins specify different capabilities to modulate B cell growth. J Virol. 2014;88:5706-17.

10. Popovic M, Tenner-Racz K, Pelser C, Stellbrink HJ, van Lunzen $\mathrm{J}$, Lewis G, et al. Persistence of HIV-1 structural proteins and glycoproteins in lymph nodes of patients under highly active antiretroviral therapy. Proc Natl Acad Sci USA. 2005;102:1480712.

11. Fiorentini S, Riboldi E, Facchetti F, Avolio Manuela, Fabbri Marco, Tosti Giorgio, et al. HIV-1 matrix protein p17 induces human plasmacytoid dendritic cells to acquire a migratory immature cell phenotype. Proc Natl Acad Sci USA. 2008;105:3867-72. 
12. Fiorentini S, Giagulli C, Caccuri F, Magiera AK, Caruso A. HIV1 matrix proteinp17: a candidate antigen for therapeutic vaccines against AIDS. Pharmacol Ther. 2010;128:433-44.

13. Tozzi V, Balestra P, Serraino D, Bellagamba R, Corpolongo A, Piselli $\mathrm{P}$, et al. Neurocognitive impairment and survival in a cohort of HIV-infected patients treated with HAART. AIDS Res \& Human Retrovir.. 2005;21:706-13.

14. Sevigny JJ, Albert SM, McDermott MP, Schifitto G, McArthur JC, Sacktor N, et al. An evaluation of neurocognitive status and markers of immune activation as predictors of time to death in advanced HIV infection. Arch Neurol. 2007;64:97-102.

15. Tozzi V, Balestra P, Bellagamba R, Corpolongo A, Salvatori MF, Visco-Comandini U, et al. Persistence of neuropsychologic deficits despite long-term highly active antiretroviral therapy in patients with HIV-related neurocognitive impairment: prevalence and risk factors. JAIDS.. 2007;45:174-82.

16. Brew BJ, Chan P. Update on HIV dementia and HIV-associated neurocognitive disorders. Curr Neurol Neurosci Rep. 2014;14:468

17. Rempel H, Sun B, Calosing C, Abadjian Linda, Montoand Alexander, Pulliam Lynn. Monocyte activation in HIV/HCV coinfection correlates with cognitive impairment. PLoS ONE. 2013;8:e55776.

18. Crews L, Patrick C, Achim CL, Everall IanP, Masliah Eliezer. Molecular pathology of neuro-AIDS (CNS-HIV). Int J Mol Sci. 2009;10:1045-63.

19. Montoya JL, Iudicello J, Fazeli PL, Hong S, Potter M, Ellis RJ, et al. Elevated markers of vascular remodeling and arterial stiffness are associated with neurocognitive function in older HIV+ adults on suppressive antiretroviral therapy. JAIDS. 2017;74:134-41.

20. Yen Y-F, Chen M, Jen I, Lan YC, Chuang PH, Liu YL, et al. Association of HIV and opportunistic infections with incident stroke: a nationwide population-based cohort study in Taiwan. JAIDS.. 2017;74:117-25.

21. Bruce J. The difficulties of 'living while girl'. J Virus Erad. 2016;2:177.

22. Seecheran VK, Giddings SL, Seecheran NA. Acute coronary syndromes in patients with HIV. Cor Art Dis.. 2017;28:166.

23. El-Sadr WM, Mayer KH, Maslankowski L, Hoesley C, Justman J, Gai F, et al. Safety and acceptability of cellulose sulfate as a vaginal microbicide in HIV-infected women. AIDS. 2006;20:1109-16.

24. Kearns A, Gordon J, Burdo TH, Qin X. HIV-1-associated atherosclerosis: unraveling the missing link. JACC.. 2017;69:3084-98.

25. McCrary AW, Nduka CU, Stranges S, Bloomfield GS. Features of cardiovascular disease in low-income and middle-income countries in adults and children living with HIV. Curr Opin HIV/ AIDS.. 2017;12:579-84

26. De Francesco MA, Baronio M, Fiorentini S, Signorini C, Bonfanti $\mathrm{C}$, Poiesi C, et al. HIV-1 matrix protein p17 increases the production of proinflammatory cytokines and counteracts IL-4 activity by binding to a cellular receptor. PNAS.. 2002;99:9972-7.

27. Vitale M, Caruso A, De Francesco MA, Rodella L, Bozzo L, Garrafa E, et al. HIV-1 matrix protein p17 enhances the proliferative activity of natural killer cells and increases their ability to secrete proinflammatory cytokines. B J Haematol. 2003;120:337-43.

28. Marini E, Tiberio L, Caracciolo S, Tosti G, Guzman CA, Schiaffonati L, et al. HIV-1 matrix protein p17 binds to monocytes and selectively stimulates MCP-1 secretion: role of transcriptional factor AP-1. Cell Microbiol. 2008;10:655-66.

29. Giagulli C, Marsico S, Magiera AK, Bruno Rosalinda, Caccuri Francesca, Barone Ines, et al. Opposite effects of HIV-1 p17 variants on PTEN activation and cell growth in B cells. PLoS ONE. 2011;6:e17831.
30. Grogg K, Miller R, Dogan A. HIV infection and lymphoma. J Clin Pathol. 2007;60:1365-72.

31. Carbone A, Gloghini A, Caruso A, De Paoli P, Dolcetti R. The impact of EBV and HIV infection on the microenvironmental niche underlying Hodgkin lymphoma pathogenesis. Int J Cancer. 2017;140:1233-45.

32. Bohlius J, Schmidlin K, Costagliola D, Fätkenheuer G, May M, Caro-Murillo AM, et al. Incidence and risk factors of HIV-related non-Hodgkin's lymphoma in the era of combination antiretroviral therapy: a European multicohort study. Antivir Ther. 2009; $14: 1065$

33. Kowalkowski MA, Day RS, Du XL, Chan W, Chiao EY Cumulative HIV viremia and non-AIDS-defining malignancies among a sample of HIV-infected male veterans. JAIDS (1999). 2014;67:204

34. Shiels MS, Engels EA, Linet MS, Clarke CA, Li J, Hall HI, et al. The epidemic of non-Hodgkin lymphoma in the United States: disentangling the effect of HIV, 1992-2009. Cancer Epidemiol Prev Biomark.. 2013;22:1069-78.

35. Oliver NT, Chiao EY. Malignancies in women with HIV infection. Curr Opin HIV/AIDS.. 2017;12:69.

36. Aserlind A, Maguire K, Duthely L, Wennin Stefan, Potter JoNell. Women living with HIV over age of 65: cervical cancer screening in a unique and growing population. Infect Dis Obstet Gynecol. 2017;2017:2105061.

37. Mthembu NN, Mbita Z, Hull R, Dlamini Zodwa. Abnormalities in alternative splicing of angiogenesisrelated genes and their role in HIV-related cancers. HIV/AIDS.. 2017;9:77.

38. Giagulli C, D'ursi P, He W, Zorzan Simone, Caccuri Francesca, Varney Kristen, et al. A single amino acid substitution confers Bcell clonogenic activity to the HIV-1 matrix protein p17. Sci Rep. 2017;7:6555

39. Giagulli C, Magiera AK, Bugatti A, Caccuri F, Marsico S, Rusnati $\mathrm{M}$, et al. HIV-1 matrix protein p17 binds to the IL-8 receptor CXCR1 and shows IL-8-like chemokine activity on monocytes through Rho/ROCK activation. Blood. 2012;119:2274-83.

40. Zlokovic BV. Neurovascular mechanisms of Alzheimer's neurodegeneration. Trends Neurosci. 2005;28:202-8.

41. Grammas P, Martinez J, Sanchez A, Yin X, Riley J, Gay D, et al. A new paradigm for the treatment of Alzheimer's disease: targeting vascular activation. J Alzheimers Dis. 2014;40:619-30.

42. Talwar P, Silla Y, Grover S, Gupta M, Agarwal R, Kushwaha S, et al. Genomic convergence and network analysis approach to identify candidate genes in Alzheimer's disease. BMC Genom. 2014;15:199.

43. Hashimoto Y, Niikura T, Chiba T, Tsukamoto E, Kadowaki H, Nishitoh H, et al. The cytoplasmic domain of Alzheimer's amyloid- $\beta$ protein precursor causes sustained apoptosis signal-regulating kinase 1/c-Jun NH2- terminal kinase-mediated neurotoxic signal via dimerization. J Pharmacol Exp Ther. 2003;306:889902.

44. Wang X, Chen Q, Xing D. Focal adhesion kinase activates NF- $\mathrm{B}$ via the ERK1/2 and p38MAPK pathways in amyloid- $\beta$ 25-35induced apoptosis in PC12 cells. J Alzheimers Dis. 2012;32:7794.

45. Epeldegui M, Vendrame E, Martínez-Maza O. HIV-associated immune dysfunction and viral infection: role in the pathogenesis of AIDS-related lymphoma. Immunol Res. 2010;48:72-83.

46. Pepper MS, Tille J-C, Nisato R, Skobe M. Lymphangiogenesis and tumor metastasis. Cell Tissue Res. 2003;314:167-77.

47. Mazzuca P, Marsico S, Schulze K, Mitola S, Pils MC, Giagulli C, et al. Role of autophagy in HIV-1 matrix protein p17-driven lymphangiogenesis. J Virol. 2017;91:e0801-00817.

48. Magi S, Takemoto Y, Kobayashi H, Kasamatsu Masato, Akita Takahiro, Tanaka Ayako, et al. 5- Lipoxygenase and cysteinyl leukotriene receptor 1 regulate epidermal growth factor-induced 
cell migration through Tiam1 upregulation and Rac1 activation. Cancer Sci. 2014;105:290-6.

49. Wang Y, Qiu J, Luo S, Xie Xiang, Zheng Yiming, Zhang Kang, et al. High shear stress induces atherosclerotic vulnerable plaque formation through angiogenesis. Regen Biomat.. 2016; 3:257-67.

50. Kayima J, Nyakoojo W, Nakanjako D, Costa MarcoA, Longenecker ChristopherT, Simon DanielI. Acute spontaneous coronary artery thrombosis as initial presentation of HIV infection in a young man. Case Rep Cardiol. 2015;2015:342348.
51. Kulkarni M, Bowman E, Gabriel J, Amburgy T, Mayne E, Zidar DA, et al. Altered monocyte and endothelial cell adhesion molecule expression is linked to vascular inflammation in human immunodeficiency virus infection. Open Forum Infect Dis. 2016;3:ofw224.

52. Renga B, Francisci D, D'Amore C, Schiaroli Elisabetta, Mencarelli Andrea, Cipriani Sabrina, et al. The HIV matrix protein p17 subverts nuclear receptors expression and induces a STAT1dependent proinflammatory phenotype in monocytes. PLoS ONE. 2012;7:e35924. 